Ophthalmologe $2022 \cdot 119: 280-287$ https://doi.org/10.1007/s00347-021-01454-w Eingegangen: 14. Februar 2021 Überarbeitet: 8. Juni 2021 Angenommen: 17. Juni 2021 Online publiziert: 20. August 2021

(c) Der/die Autor(en) 2021

\section{Das Hamburger Register für intravitreale Injektionstherapien (QIVOM)}

\author{
Christian Wolfram ${ }^{1}$ (D) $\cdot$ Marc Schargus ${ }^{2,3}$ \\ ${ }^{1}$ Universitätsklinikum Hamburg-Eppendorf (UKE), Hamburg, Deutschland \\ ${ }^{2}$ Asklepios Augenklinik Nord-Heidberg, Hamburg, Deutschland \\ ${ }^{3}$ Klinik für Augenheilkunde, Heinrich-Heine-Universität, Düsseldorf, Deutschland
}

\title{
Zusammenfassung
}

Hintergrund: Intravitreale operative Medikamenteneingaben (IVOM) gehören zu den häufigsten medizinischen Prozeduren überhaupt mit ca. 1,5 Mio. Eingriffen in Deutschland pro Jahr. Für diese enorme Versorgungsaufgabe gibt es nur wenige empirische Daten über den Versorgungsprozess und seine klinische und subjektive Wirkung.

Material und Methoden: Es wird die Entwicklung und der Aufbau des Hamburger Registers für intravitreale Injektionstherapien (QIVOM) detailliert beschrieben. IVOMPatienten der drei großen Augenkliniken Hamburgs (Asklepios Kliniken Nord-Hamburg und Barmbek, Universitätsklinikum Hamburg-Eppendorf) werden im Rahmen der Routineversorgung mit IVOM zur Studienteilnahme eingeladen. Es werden subjektive Patientenangaben zum Krankheits- und Behandlungserleben erhoben und diese mit den patientenbezogenen medizinischen Parametern elektronisch ergänzt, pseudonymisiert und in einer elektronischen Datenbank erfasst.

Ergebnisse: Von den ersten 162 Studienpatienten (Alter 41-95 Jahre) war die Behandlungsindikation bei $64 \%$ exsudative altersbedingte Makuladegeneration (AMD), bei 22\% retinaler Venenverschluss und bei 11\% diabetisches Makulaödem. Es ergibt sich ein heterogenes Bild der Erkrankungsschwere und der subjektiven Beeinträchtigung. Eine Sehschärfe von 0,5 und besser wiesen 31,8\% der Patienten am behandelten Auge im Vergleich zu 79,1\% am Partnerauge auf. Die größte praktische Einschränkung betraf die Lesefähigkeit, bei der über ein Drittel erhebliche Einschränkungen erlebte. Autofahren war immerhin für $62 \%$ möglich. Eine Verbesserung der Sehfähigkeit durch IVOM erreichten $45 \%$ der Patienten.

Schlussfolgerung: Die Datenerfassung von patientenseitigen Daten und medizinischer Versorgungsdaten aus den Kliniken stellt den großen Vorteil des neu geschaffenen Registers dar. Die Erweiterung der Datenbasis soll in Zukunft vielfältige weiterführende Erkenntnisse erbringen und zur Qualitätssicherung in der IVOM-Therapie beitragen.

\section{Schlüsselwörter}

Register · Intravitreale Therapie · Anti-VEGF-Antikörper · Patientenorientierung · Deutschland

\section{Hintergrund und Fragestellung}

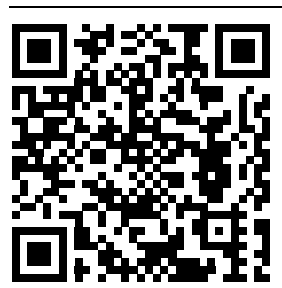

QR-Code scannen \& Beitrag online lesen
Die Diskrepanz der Ergebnisse klinischer Studien und tatsächlich erreichter klinischer Resultate im Versorgungsalltag hat die Erforschung der Behandlungswirklichkeit („,real life") in den letzten Jahren in den Fokus gerückt. Für verschiedene ophthalmologische Erkrankungen wurden Krankheitsregister aufgebaut, um die Verbrei- tung und spezifische Versorgung abzubilden [15].

Intravitreale operative Medikamenteneingaben (IVOM) gehören mittlerweile zu den häufigsten medizinischen Eingriffen überhaupt. Für Deutschland berichten Wenzel et al. [22] in ihrer jährlichen Um- 
Tab. 1 Subjektive Parameter für intravitreale Injektionstherapien aus Patientenperspektive

Angaben zum Gesundheits- und Krankheitserleben

- Wahrnehmung der Symptome der Netz hauterkrankung

- Bisheriges Krankheitserleben

- Ein- oder Beidseitigkeit der Erkrankung

- Begleiterkrankungen

- Dauermedikation

- Alltagsfähigkeiten (Autofahren, Lesen, Erkennen von Menschen)

- Erfassung von Gesundheitserleben und Lebensqualität (Fragebogen EQ-5D [4])

- Psychosoziale Versorgung

- Erfassung klinisch symptomatischer Angstzustände (Fragebogen GAD-2 [24])

- Initiale Therapieerwartungen

- Bewertung der bisherigen Injektionstherapien auf das Sehvermögen

Erleben der medizinischen Versorgung

- Erleben des bisherigen Behandlungsverlaufs

- Zeitraum zwischen Diagnosestellung und Erstinjektion

- Häufigkeit von OCT-Kontrollen

- Anzahl bisheriger Injektionstherapien

- Therapieintervall

- Bewertung der Häufigkeit und Schwere von Nebenwirkungen

- Patientenzufriedenheit

- Therapieadhärenz (Unterbrechungen, Abbrucherwägungen)

- Anzahl der Arztbesuche

- Persönlicher Aufwand (Anfahrt, Wartezeit, Begleitung)

Soziodemografische Angaben

- Alter

- Geschlecht

- Schul- und Berufsabschluss

- Beruflicher Status

- Familienstand

- Haushaltsstatus

- Pflegebedürftigkeit

frage zur ambulanten Okularchirurgie ${ }^{1}$ über eine Zahl von 497.719 erfassten IVOM-Therapien für 2019, wobei nur 24\% der versendeten Umfragebögen ausgefüllt zurückgeschickt wurden (38\% Rücklaufquote unter ophthalmologischen

1 Beteiligt sind die Augenverbände BDOC, BVA, DGll und DOG.
Tab. 2 Klinische Parameter (aus der Patientenakte)

\begin{tabular}{l} 
- Zentrale Sehschärfe (Dezimalvisus) \\
\hline - Behandlungsindikation \\
\hline - Ophthalmologische Komorbiditäten \\
- Refraktionsstatus \\
- Linsenstatus \\
- Augeninnendruck \\
- Systemische Komorbiditäten \\
- Bei Diabetikern: Dauer, Blutzuckereinstel- \\
lung (HbA1c), Insulinpflicht \\
- Psychologische Komorbiditäten \\
- Erkrankungsdauer \\
- OCT-Befund (zentrale Netzhautdicke in \\
Mikrometern am primär behandelten \\
und am Partnerauge) \\
- Therapieschema \\
- Therapieverlauf
\end{tabular}

Hauptabteilungen) und nicht alle Versorgungsstellen erfasst sind, in denen IVOM-Therapien durchgeführt werden. Vorsichtig geschätzt kann man also von einer mindestens dreifachen Häufigkeit der erfassten Zahlen ausgehen, was somit mindestens 1,5 Mio. jährlichen Injektionen für Deutschland entspricht. Trotz dieser enormen Verbreitung als medizinischem Eingriff ist nur relativ wenig über die Versorgungspraxis und die klinische und subjektive Wirkung der Therapien bekannt. So stellt sich etwa der Unterschied zwischen intendierter und erreichter Versorgung besonders dar: Während die großen Zulassungsstudien für Anti-VEGFMedikamente bis zu 12 Injektionen pro Jahr erzielten $[8,10,11]$, wurden in Beobachtungsstudien des Versorgungsalltags nur 4 bis 5 Injektionen pro Jahr erreicht $[2,20,25]$. Weiterhin wurde beschrieben, dass eine höhere Injektionsfrequenz zu besseren Visusergebnissen führte [7, 21]. Um die Diskrepanz zum Versorgungsalltag zu verstehen, ist es notwendig, durch weiterführende Studien (vgl. auch [5]) mehr Informationen über die Perspektive des Patienten, sein Krankheitserleben und sein Verhalten zu erfahren, die Versorgungsabläufe und -routinen näher zu beschreiben und zu hinterfragen, welche klinische und subjektive Wirkung IVOMTherapien im Alltag tatsächlich erzielen.

Das Hamburger Register für intravitreale Injektionstherapien (QIVOM) soll eine indikationsübergreifende Datenbasis über den Versorgungsalltag bei intravitrealen Injektionen schaffen. Diese dient dem Zweck, sowohl die Strukturen und Prozesse als auch die klinischen und subjektiven Ergebnisse von IVOM-Therapien zu beschreiben und zu analysieren. Dieser Artikel stellt den Aufbau und die Struktur des Registers vor und präsentiert erste Studienergebnisse.

\section{Studienkonzeption}

Die Datenerhebung für das Hamburger Register für intravitreale Injektionstherapien (QIVOM) geht vom Patienten und seiner Erfahrung der zugrunde liegenden Netzhauterkrankung und Versorgungserfahrung aus. Dazu wurde nach einer qualitativen Vorstudie mit zehn Patienteninterviews ein Registerprotokoll entwickelt, das sich an den Vorgaben der Deutschen Netzwerks für Versorgungsforschung [12, 19] orientiert. Es werden subjektive Informationen und Bewertungen durch den Patienten selbst erfasst und ergänzend medizinische Parameter aus der Patientenakte entnommen. Die verschiedenen Parameter sind in 0 Tab. 1 und 2 zusammengefasst.

Insgesamt werden bis zu 69 Fragen vom Patienten beantwortet, dazu gehören neben den Angaben zum Krankheitsverlauf auch neun Fragen zur soziodemografischen Situation. Weiterhin besteht auch die Möglichkeit, offene Kommentare einzufügen. Aus der Patientenakte werden darüber hinaus bis zu 24 weitere klinische Parameter berücksichtigt. Die unterschiedliche Anzahl der Fragen und Parameter ergibt sich, da für besondere Patientengruppen wie Patienten mit Diabetes zusätzliche Items abgefragt werden. Bei den Verlaufsuntersuchungen sind weniger Fragen vom Patienten zu beantworten, und es müssen auch weniger medizinische Informationen aus der Patientenakte extrahiert werden. Im weiteren Verlauf der Registererhebung kann die Datenerhebung modulweise erweitert oder gekürzt werden, wenn sich z. B. besondere neue Fragestellungen ergeben oder falls einzelne Fragen oder Parameter nicht mehr benötigt werden.

Die jeweiligen Fragenkataloge und einzelnen Antwortmöglichkeiten werden als Electronic Case Report Forms (ECRF) für die teilnehmenden Studienzentren auf einer 


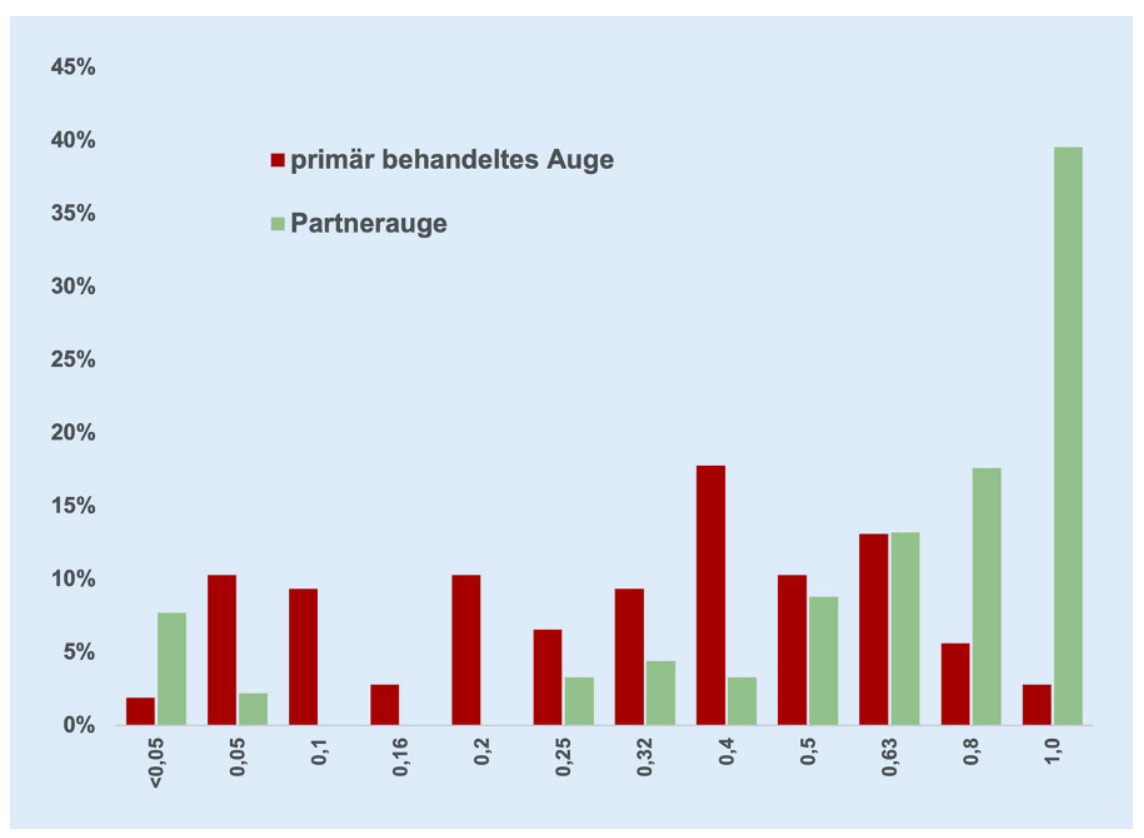

Abb. 1 \ Prozentuale Verteilung des Dezimalvisus am primär behandelten Auge (rot) und am Partnerauge ( $g r u ̈ n)$ in 12 Stufen

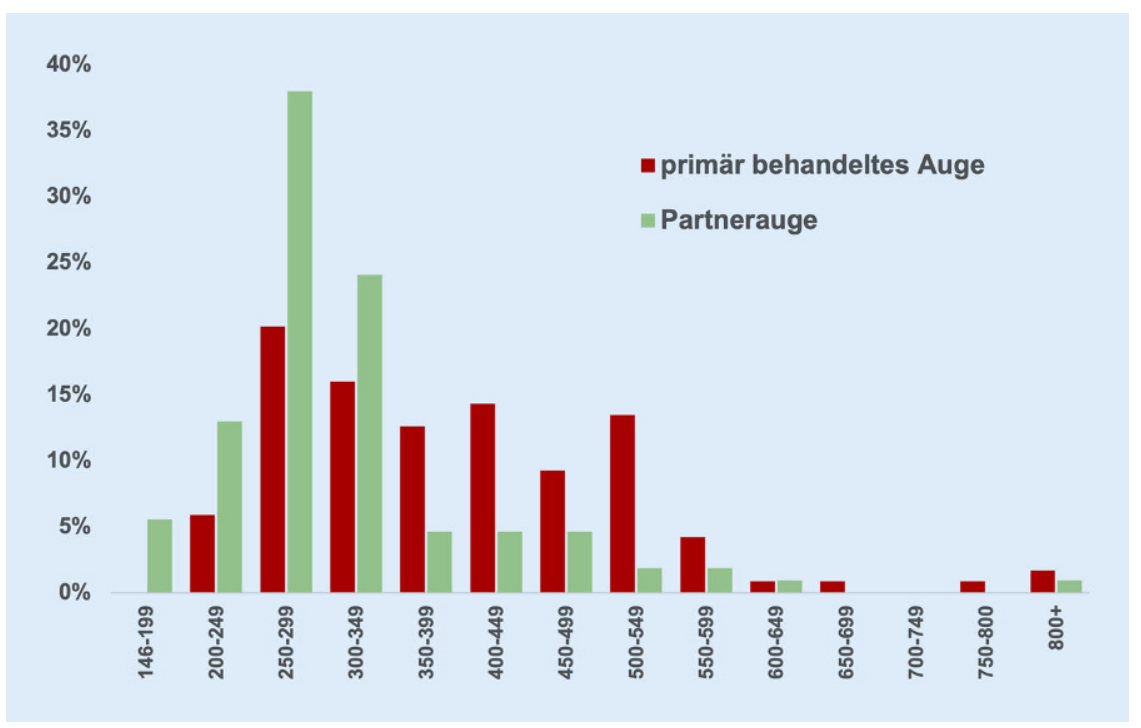

Abb. $2 \Delta$ Prozentuale Verteilung der zentralen Netzhautdicke in Mikrometer $(\mu \mathrm{m})$ am primär behandelten Auge (rot) und am Partnerauge (grün) in 14 Stufen

eigenen Webseite (www.qivom.de) veröffentlicht.

\section{Patienteneinschluss}

Es werden derzeit Patienten in die Datenerhebung eingeschlossen, die sich einer intravitrealen Therapie aufgrund einer Netzhauterkrankung unterziehen an einer der Hamburger Augenkliniken Asklepios Nord-Heidberg, Asklepios Barmbek oder dem Universitätsklinikum Eppendorf ligen Fragen verstehen und beantworten zu können.

\section{Datendokumentation}

Die medizinischen Daten werden anschließend aus der elektronischen Patientenakte (für Asklepios Nord-Heidberg und Asklepios Barmbek FIDUS, Darmstadt, für die Universitäts-Augenklinik Hamburg-Eppendorf ifa systems, Frechen) ergänzt. Die patientenseitigen Angaben und die medizinischen Daten werden in jeweils einem elektronischen Formular (Electronic Case Report Forms - ECRF) pseudonymisiert auf einen zentralen Server, der sich auf dem UKE-Gelände befindet, hochgeladen. Da die Patientendaten bereits bei der Übertragung in die zentrale Datenbank nicht personalisiert sind, ist eine Rückverfolgung zum Einzelnen aus den Registerdaten nicht möglich, sodass ein größtmögliches Level an Datenschutz für die Patienten gewährleistet ist. Der Zugriff auf die Studiendaten ist nur für autorisiertes Studienpersonal möglich. Längerfristig soll eine wissenschaftlicher Beirat eingerichtet werden, der über den Datenzugriff und die jeweilige Datenanalyse entscheidet. Auf diese Weise soll die wissenschaftliche Unabhängigkeit der Registeranalysen gewahrt bleiben.

Die technische Infrastruktur des Registers wurde gemeinsam mit dem SoftwareAnbieter Swiss4ward (Zürich, Schweiz) entwickelt, der sich auf die Entwicklung von medizinischen Registern und elektronischen Datenbanken spezialisiert hat. Zwei positive Ethikvoten von der zuständigen Ethikkommission der Ärztekammer Hamburg wurden für die bisherigen Studienprojekte eingeholt. (Ethikvoten der Ärztekammer Hamburg: PV7208/PV7202).

Zur Illustration der Registerinhalte stellen wir die Ergebnisse einer ersten explorativen Querschnittsanalyse des bisherigen Studienkollektivs vor. Dazu wurden aus den Angaben durch die Patienten sowie aus den vorliegenden medizinischen Daten bei kategorialen Variablen einfache Häufigkeiten errechnet. Im Fall stetiger Variablen wurden Mittelwerte errechnet. 


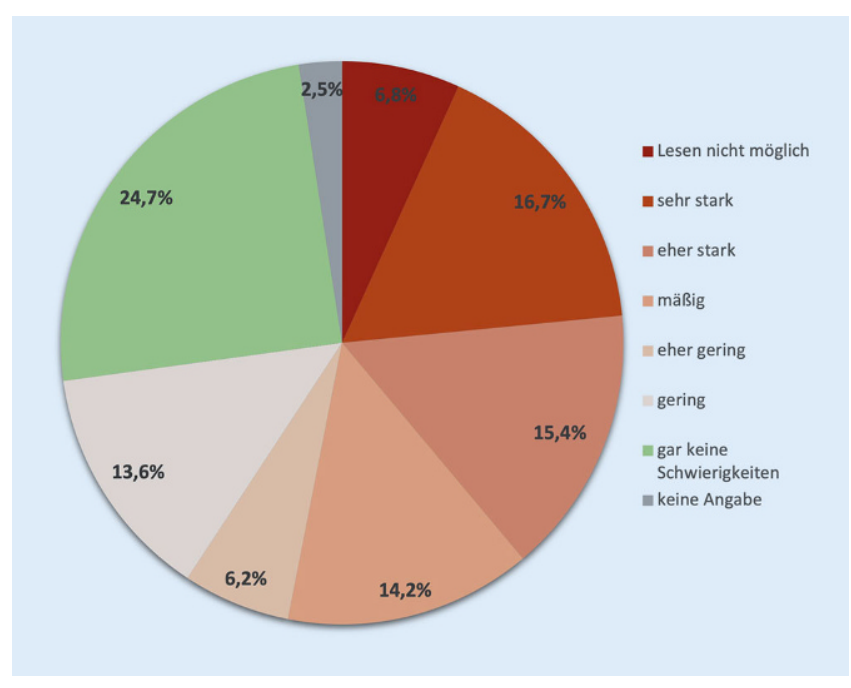

Abb. $3 \triangleleft$ Verteilung der Lesefähigkeit unter IVOMTherapie („Haben Sie Schwierigkeiten beim Lesen, z. B. der Zeitung?")

\section{Ergebnisse}

\section{Studienkollektiv}

In unserer Auswertung lagen subjektive Angaben von 162 Patienten und klinische Informationen aus der Patientenakte von 140 Patienten vor, die sich im Zeitraum zwischen Dezember 2019 und Dezember 2020 in einer der genannten Hamburger Augenkliniken einer IVOM-Therapie unterzogen haben. Das Alter der Studienteilnehmerinnen und -teilnehmer lag zwischen 41 und 95 Jahren (Durchschnitt 75,4 Jahre) bei einer Verteilung nach Geschlechtern von $61 \%$ weiblichen $(n=85)$ und $39 \%$ männlichen Patienten $(n=55)$. Bei $76 \%$ der Patienten erfolgte die Therapie an einem Auge $(n=107)$, bei $24 \%$ $(n=33)$ an beiden Augen. Die Therapieindikation im Patientenkollektiv war in $64 \%$ der Fälle $(n=90)$ eine exsudative AMD, bei $22 \%(n=31)$ ein retinaler Venenverschluss und bei $11 \%(n=15)$ ein diabetisches Makulaödem. Nach Patientenangaben wurde in $65 \%$ der Fälle $(n=106)$ ein vierwöchiges Therapieschema verfolgt, bei $15 \%(n=25)$ ein unregelmäßiges und in $12 \%(n=22)$ ein 6- oder mehrwöchiges Schema (ohne Angabe 5\%; $n=9$ ). Nach Angaben aus der Patientenakte verfolgten $16 \%(n=22)$ ein initiales monatliches Pro-re-nata(PRN)-Therapieschema, weitere $30 \%(n=42)$ waren im fortgesetzten monatlichen PRN-Schema und sogar $49 \%$ ( $n=69)$ im Treat\&Extend(TE)-Schema. Bei $5 \%(n=7)$ lag keine Angabe zum Therapieschema vor.

\section{Verteilung von Visus und zentraler Netzhautdicke}

Die Sehschärfe am primär behandelten $\mathrm{Au}$ ge war in 31,8\% der Fälle 0,5 und besser, am Partnerauge hingegen $79,1 \%$ (• Abb. 1). Analog dazu war der Anteil derjenigen Patienten mit einer zentralen Netzhautdicke von weniger als $350 \mu \mathrm{m}$ am primär behandelten Auge nahezu halb so groß wie am Partnerauge (42 vs. $80 \%$, Abb. 2 ). ${ }^{2}$

\section{Subjektives Erkrankungserleben und funktionelle Einschränkungen}

Im Studienkollektiv berichteten $45 \%$ der Patienten von einer schleichenden und $44 \%$ von einer plötzlichen Sehminderung, diezum Behandlungsbedarffür eine IVOMTherapie geführt habe. $13 \%$ der Patienten gaben an starke Schwierigkeiten beim Erkennen von Menschen zu haben, für $2 \%$ war das sogar unmöglich. Dahingegen hatten weitere $13 \%$ geringe und $59 \%$ gar keine Schwierigkeiten. Lesen dagegen war für 32\% stark eingeschränkt und für 7\% der Patienten sogar unmöglich, wohingegen bei $44 \%$ nur geringe oder gar keine Schwierigkeiten bestanden (- Abb. 3).

$62 \%$ gaben an, noch Auto fahren zu können, gegenüber $36 \%$, die dieses nicht taten. Davon nannten 15\%, dass ihr Sehvermögen dafür nicht ausreiche, $12 \%$ führ-

2 Die Streuungsbreite bei den Angaben der Partneraugen ergibt sich, da hier auch die Fälle beidseitig therapierter Augen berücksichtigt sind. ten andere gesundheitliche Gründe an, und $9 \%$ hatten keinen Führerschein. Die lebenspraktischen Beschwerden bestanden für die Betroffenen demnach eher im Lesen als im Erkennen von Menschen oder in der Fahrfähigkeit.

Für die Bewertung der Lebensqualität stellte eine Hälfte der Patienten (50\%) keine oder nur geringe Auswirkungen der Netzhauterkrankung auf die Lebensqualität fest, wohingegen $23 \%$ diese Lebensqualität als „einigermaßen“ und $27 \%$ als stark bis sehr stark beeinträchtigt bewerteten.

\section{Patientenerwartungen und Therapieergebnisse}

Rückblickend haben zwei Drittel der Befragten bei Therapiebeginn eine Verbesserung durch die Injektionstherapie erhofft. Diese wurde bis zum Zeitpunkt der Untersuchung auch bei $45 \%$ nach eigenen Angaben erreicht, jedoch haben etwa $17 \%$ im Verlauf eine Verschlechterung ihrer Sehfähigkeit erlebt (• Abb. 4).

\section{Diskussion}

Das Hamburger Register für intravitreale Injektionen (QIVOM) ermöglicht eine detaillierte Abbildung der Versorgungssituation von Netzhauterkrankungen, die durch intravitreale Injektionstherapien (IVOM) behandelt werden. Konzipiert als längerfristige Registerstudie sollen indikationsübergreifende Erkenntnisse über die IVOM-Versorgung gewonnen und beschrieben werden.

Die ersten explorativen Ergebnisse offenbaren eine große Heterogenität hinsichtlich der Erkrankungsschwere und der Auswirkungen auf die Lebensqualität bei den betroffenen IVOM-Patienten. Während die Mehrheit der behandelten Patienten über einen funktionell relativ guten Visus verfügt und sowohl durch die Therapie als auch durch das Sehen des Partnerauges nur relativ geringe lebenspraktische Einschränkungen erlebt, ergibt sich für etwa ein Drittel der versorgten Patienten ein erheblicher Einfluss auf die subjektive Sehfunktion und Lebensqualität. Naheliegend ist, dass es bei so unterschiedlichen Ausgangspositionen auch deutliche Unterschiede gibt für die Bereitschaft, sich 


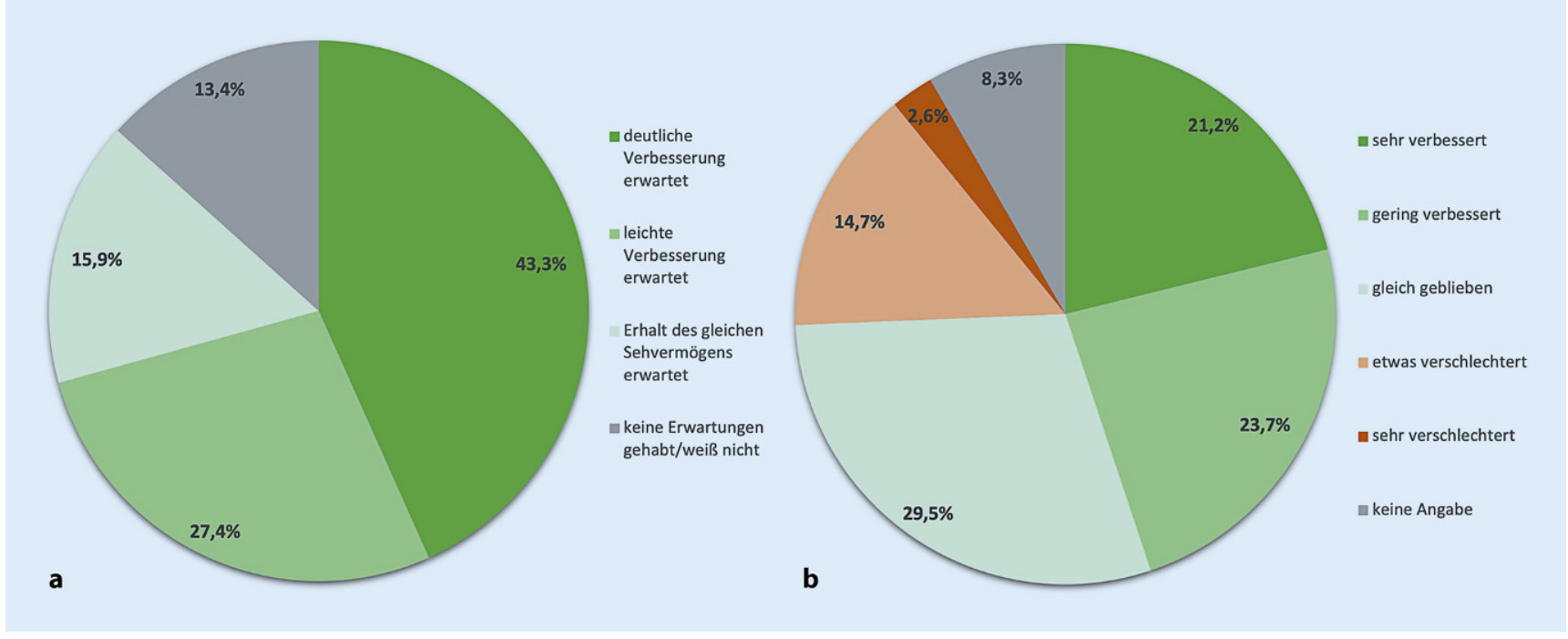

Abb. 4 \ Erwartete Visusentwicklung vor Therapiebeginn (a) und tatsächliche Visusentwicklung im Therapieverlauf (b)

dauerhaft der Therapie zu unterziehen. So wies die AURA-Studie in einer zweijährigen Beobachtungszeit nach, dass ein guter Ausgangsvisus und ein niedrigeres Lebensalter zu Beginn der IVOM-Therapie sowie eine höhere Therapieadhärenz mit einer besseren Visusprognose einhergingen [7]. Longitudinale Untersuchungen des Patientenverhaltens und des Behandlungsverlaufs können die Wechselwirkung zwischen subjektiver Sehfunktion und der Therapieadhärenz weiter ergründen mit dem Ziel, einen vorzeitigen Abbruch von Therapien zu vermeiden $[5,9]$.

Patienten nehmen die Injektionstherapie sehr unterschiedlich wahr. Bekannt ist, dass viele Patienten Ängste und Schmerzen im Zusammenhang mit der Injektion erleben. In einer qualitativen Untersuchung gaben $56 \%$ der Patienten Ängste an, nach einem klinischen Score für Angst waren $17 \%$ betroffen [17]. In einer anderen Studie lag eine klinisch relevante Angstprävalenz bei $25 \%$ der Patienten vor [16]. Auch für Schmerzen wurden bei $58 \%$ der Patienten in irgendeiner Form Schmerzen bei der Injektion angegeben, wenngleich diese in weit überwiegendem Maß geringfügig und nur für etwa $5 \%$ in klinisch höherem Ausmaß bewertet wurden [14]. Die Beschreibung und Analyse solcher Patientenerfahrungen ist hilfreich, um Hindernisse im Versorgungsweg gezielter verstehen und adressieren zu können. Die Analyse der psychosozialen Begleiterkrankungen, des allgemeinen Gesundheitszustands und der spezifischen Bewertung der Behandlungssituation kann das Verständnis der patientenseitigen Wahrnehmung der IVOM-Therapie vertiefen und ggf. einen besonders vorsichtigen Therapiezugang für sensiblere Patientengruppen anstoßen.

Auffällig ist auch der Unterschied in unseren Ergebnissen zwischen der subjektiven Angabe, nach der sich $65 \%$ der Patienten in einem monatlichen Therapieschema befinden, gegenüber nur $46 \%$, bei denen gemäß der Patientenakte ein monatliches PRN-Schema verfolgt wird. Anzunehmen ist demnach, dass die Dokumentation eines Treat\&Extend-Schemas von Patienten nicht immer als Veränderung des Behandlungsintervalls wahrgenommen wird. Mehr Erkenntnisse über den Wissens- und Informationsstand von Patienten können helfen, mögliche Diskrepanzen zwischen intendierter und erreichter Versorgung zu erklären.

Erfreulicherweise stellen $45 \%$ der Patienten eine subjektive Visusverbesserung unter der IVOM-Therapie fest, wenngleich die Erwartung vor Therapiebeginn nach eigener Auskunft noch höher war. Für die Kontinuität der Therapie über einen längeren Zeitraum ist es wichtig, ob Patienten den Verlauf ihrer Therapie als Erfolg oder als Misserfolg deuten. Die hohe Rate von Nonadhärenz bei der IVOM-Therapie aus anderen Erhebungen - bei AMD bis $32 \%$ und bei Diabetikern sogar bis $44 \%$ [3] deuten darauf hin, dass es nicht immer ausreichend gelingt, die Patienten von der
Therapiefortsetzung zu überzeugen. Therapieabbrüche wurden in der Vergangenheit mit Unzufriedenheit bei etwa einem Drittel oder einer zu intensiven Therapie bei einem Viertel der Betroffenen begründet [6] oder auch mit zu hohen und unrealistischen Therapieerwartungen [18]. Gerade für diejenigen Patienten, die trotz Therapie eine Verschlechterung erfahren - in unserer Untersuchung immerhin 17\%-, stellt sich die Frage der weiteren Therapiemotivation und -fortführung besonders. Mehr Wissen über Diskontinuitäten und Nonadhärenz in der Therapie kann daher dazu beitragen, Versorgungs- und Vertrauensmängel genauer zu identifizieren und die zukünftige Versorgung zu verbessern.

\section{Ausblick Versorgungsmonitoring}

Das hier skizzierte Versorgungsregister für intravitreale Injektionstherapien verfügt über die nötige konzeptionelle und technische Infrastruktur, um auch kontinuierliche Daten über das Versorgungsgeschehen bei IVOM-Therapien zu erheben. Das Ziel ist es, die Datenerfassung über einen längeren Verlaufszeitraum auszudehnen und zu vergrößern, sodass das Register ein wichtiges Instrument zum Monitoring der Versorgung auf mehreren Ebenen werden kann. So ist zunächst auf der Ebene des Patienten die subjektive Bewertung des Krankheitsverlaufs den medizinischen Verlaufsdaten gegenüberzustellen und zu hinterfragen, inwiefern die Bereitschaft 
Tab. 3 Auflistung longitudinaler Merkmale und Ziele des Registers

- Injektionsfrequenz

- Individuelle Versorgungsverläufe

- Analyse der Therapieadhärenz

- Untersuchung von Therapieabbrüchen und ihren Gründen

- Beschreibung und Erforschung von längerfristigen Versorgungstrends

- Qualitätsberichte für Patienten und Versorger

- Identifikation von Versorgungsunterschieden nach Sektoren/Einrichtungen/ Regionen

- Identifikation von unterversorgten Patientengruppen

- Unterstützung klinischer und epidemiologischer Forschung

- Evaluation und Förderung der Patientensicherheit

- Pharmakovigilanzanalysen

- Marktanalysen

- Gesundheitsökonomische Studien

- Unterstützung der Versorgungsplanung

der Patienten, sich der IVOM-Therapie zu unterziehen, im längeren Verlauf der Therapie aufrechterhalten bleibt. Auch die Frage, ob sich die Therapieadhärenz je nach Krankheitsschwere, visueller Funktion oder bei beidäugiger Therapie im Kontrast zu einseitiger Behandlung unterschiedlich darstellt, sollte ebenso Gegenstand weiterer Analysen sein wie Fragen nach indikations-, alters- oder geschlechtsspezifischen Unterschieden. Gerade die Erforschung von Therapieabbrüchen bietet die Chance, das Patientenverhalten besser verstehen zu lernen und so Diskontinuitäten noch gezielter entgegenwirken zu können.

Als Instrument der Qualitätssicherung in der IVOM-Therapie ergeben sich aus den Registerdaten auch wichtige Rückschlüsse auf der Ebene der Behandler. So geben Informationen über die Patientenzufriedenheit oder die Wahrnehmung der Abläufe der IVOM-Therapie eine Rückmeldung zur Versorgungspraxis. Konkrete Angaben zur erreichten Injektionsfrequenz, aber auch zur Häufigkeit von schwerwiegenden Nebenwirkungen sind zudem wichtige Parameter der Versorgungsqualität und des Versorgungsmanagements.

Nicht zuletzt auch auf der wissenschaftlichen und gesellschaftlichen Ebene er- möglichen Registerdaten, größere Versorgungsmuster und längerfristige Trends zu beschreiben und zu analysieren (•Tab. 3).

Krankheitsregister haben in der deutschen Augenheilkunde bislang noch keine lange Tradition [15], was umso erstaunlicher ist, da es in Deutschland aufgrund der föderalen Länder- und Sozialversicherungsstrukturen kaum nationale Daten oder valide Routinedatenerhebungen gibt. Staatliche Gesundheitssysteme wie in Schweden können relativ einfach Versorgungsdaten aus einer nationalen Datenbank extrahieren und so etwa die durchschnittliche Injektionsfrequenz (5,9 Injektionen pro Jahr für 2014 für die exsudative AMD) errechnen [23]. Naheliegend wäre es daher, aus medizinischen Routinedaten (z. B. Krankenkassendaten) ähnliche Erkenntnisse für Deutschland ziehen zu wollen. Dieser Zugang erweist sich jedoch als wenig praktikabel, da solche Routinedaten Fehlklassifikationen unterliegen (u.a. durch Überdokumentationen erlösrelevanter Leistungen [13]) und diese zudem keine klinischen Informationen wie z.B. den OCT-Befund oder den Visus abbilden. Ungenaue Klassifikationen, wie z. B. die fehlende Differenzierung der ICDCodes für exsudative Makulaerkrankungen, die erst im Jahr 2020 geändert wurden, tragen zu dem Problem der zentralen Auswertung zusätzlich bei. Eine höhere externe Validierung und eine detailliertere Beschreibung von Krankheitsverläufen, Komorbiditäten und Behandlungsregimes ermöglicht daher einen Erhebungsansatz aus der Alltagsversorgung, der vom Patienten und seinem Krankheitsleben ausgeht und subjektive und objektive klinische Angaben kombiniert (vgl. [1]).

Der Erkenntnisgewinn der Registerforschung zielt ab auf den Nachweis der Wirksamkeit (Effectiveness) im Versorgungsalltag, die anders ist als Wirkprinzipien und Wirkungen (Efficacy), wie sie randomisierte kontrollierte klinische Studien hervorbringen. Die Antwort einer Versorgungsstudie ist also weniger auf die Frage ausgerichtet, wie ein Therapieverfahren wirkt, als vielmehr darauf, ob und wie dieses im Versorgungsalltag erfolgreich ist. Klinische Studien sind charakterisiert durch eine Limitierung auf selektionierte Patientengruppen und enge Ein- und Ausschlusskriterien. Bei Registerstudien hingegen ist die Zahl der
Patienten grundsätzlich nicht limitiert und schließt somit auch heterogene Patientengruppen und weitere Studienzentren ein. Weiterhin werden verschiedene Therapieverfahren und Behandlungskonzepte berücksichtigt, womit idealerweise eine größere Repräsentativität oder externe Validierung erreicht wird [13]. Eine weit höhere Zahl der eingeschlossenen Patienten und der Studienzentren (gerade auch im niedergelassenen Bereich) kann die Aussagekraft der Ergebnisse verbessern, da theoretisch bei einer einseitigen und zu geringen Patientenzahl die Abbildung der Versorgungswirklichkeit auch verzerrt sein kann. Da wir uns in unserer Untersuchung auf stationäre Einrichtungen und die urbane Situation in Hamburg beziehen, ist ein solcher Selektionsbias nicht auszuschließen, wenn es um den Rückschluss auf die Versorgung der Gesamtpopulation geht. Da die untersuchten IVOM-Behandlungen in den Kliniken jedoch alle ambulant durchgeführt wurden, ist anzunehmen, dass die Versorgungsroutinen und Behandlungsregime sich nicht grundlegend unterscheiden vom ambulanten Versorgungssektor. Bei einer größeren Anzahl der untersuchten Patienten könnten dennoch Versorgungsunterschiede zwischen den Versorgungssektoren und auch zwischen einzelnen Einrichtungen ausfindig gemacht werden. Ebenso könnte der Vergleich der Versorgung z. B. mit entlegeneren Landgegenden regionale Versorgungsprobleme genauer identifizieren.

Somit sind die Erkenntnisse aus Registeranalysen in mehrfacher Hinsicht wertvoll: für Versorger im Sinne einer internen Qualitätssicherung und möglicherweise auch eines Benchmarks, für den augenärztlichen Berufsstand, indem die gesundheitsökonomische und gesundheitspolitische Relevanz dieses Versorgungsbereichs verdeutlicht wird, für die Versorgungsplanung durch ein regelmäßiges Monitoring der Versorgungssituation und die Möglichkeit, Versorgungskonzepte zu optimieren, und nicht zuletzt auch für die Patienten selbst für die Wahrung der Patientensicherheit, der Therapieadhärenz und der Zufriedenheit mit der IVOM-Therapie. Eine wirklichkeitsgetreue Abbildung der Versorgungssituation im Register kann somit dazu beitragen, den betroffenen Patientinnen und Patienten realistische Erwar- 
tungen für die eigene IVOM-Therapie zu vermitteln.

\section{Fazit für die Praxis}

- Das Hamburger Register für intravitreale Injektionstherapien (QIVOM) soll eine indikationsübergreifende Datenbasis zur Abbildung und Beschreibung der Behandlungswirklichkeit von IVOM-Therapien schaffen.

- Das QIVOM-Register kombiniert subjektive Angaben vom Patienten mit medizinischen Daten aus der Patientenakte.

- Die longitudinale Registererhebung soll zum längerfristigen Monitoring und zur Qualitätssicherung in der IVOM-Therapie beitragen.

- Die erste explorative Querschnittsanalyse zeigt, dass das klinische Bild von Patienten, die sich einer IVOM-Therapie unterziehen, hinsichtlich Visus und OCT-Befund sehr heterogen ist. Auch die persönliche Bewertung des Krankheitserlebens und der Lebensqualität unterscheiden sich erheblich.

- Mehrheitlich gelingt Patienten ein Leben ohne größere Einschränkungen, etwa ein Drittel der Patienten erlebt hingegen schwerere praktische Limitationen.

Korrespondenzadresse

Priv.-Doz. Dr. med. Christian Wolfram, MPH, FEBO

Universitätsklinikum Hamburg-Eppendorf

(UKE)

Martinistraße 52, 20246 Hamburg, Deutschland c.wolfram@uke.de

Funding. Open Access funding enabled and organized by Projekt DEAL.

\section{Einhaltung ethischer Richtlinien}

Interessenkonflikt. C. Wolfram und M. Schargus geben an, dass das Registerprojekt QIVOM derzeit gefördert wird durch Forschungsgelder von Novartis Pharma sowie von Asklepios Proresearch.

Für diesen Beitrag wurden von den Autoren keine Studien an Menschen oder Tieren durchgeführt. Für die aufgeführten Studien gelten die jeweils dort angegebenen ethischen Richtlinien.

Open Access. Dieser Artikel wird unter der Creative Commons Namensnennung 4.0 International Lizenz veröffentlicht, welche die Nutzung, Vervielfältigung, Bearbeitung, Verbreitung und Wiedergabe in jeglichem Medium und Format erlaubt, sofern Sie den/die ursprünglichen Autor(en) und die Quelle ordnungsgemäß nennen, einen Link zur Creative Commons Lizenz beifügen und angeben, ob Änderungen vorgenommen wurden.

\section{The Hamburg register for intravitreal injection therapies (QIVOM)}

Background: Intravitreal drug delivery belongs to the most common medical procedures, with approximately 1.5 million treatments per year in Germany. However, for this enormous challenge to provision of proper care, there is a lack of empirical data regarding the treatment process and its clinical and subjective effect.

Materials and methods: This publication presents the development and structure of the Hamburg register for intravitreal therapies (QIVOM). Patients undergoing intravitreal injection therapy were invited to join the register study. Patients were recruited from the Hamburg eye clinics in Heidberg-Nord and Barmbek as well as from the University Medical Center Hamburg-Eppendorf (UKE). Both subjective patientreported information about the perception of their eye disease and its treatment as well as medical parameters were stored in a central electronic database.

Results: Of the first 162 study patients (aged 41-95 years), 64\% suffered from wet age-related macular degeneration (AMD), $22 \%$ had a retinal vein occlusion, and $11 \%$ a diabetic macula edema. Disease severity and subjective impairment were heterogenous. Among these patients, $31.8 \%$ had a visual acuity above $10 / 20$ on the treated eye compared to $79.1 \%$ on the other eye. The reduced ability to read was the most relevant limitation for more than one third of patients. However, $62 \%$ were able to drive a vehicle. An improvement of vision through intravitreal therapy was experienced by $45 \%$.

Conclusion: Collection of patient-reported as well as treatment-related data comprises the advantage of the newly created register. This extension of the data basis should deliver new findings in the future and contribute to quality assurance in intravitreal care.

\section{Keywords}

Register $\cdot$ Intravitreal injections · Anti-VEGF antibodies $\cdot$ Patient orientation $\cdot$ Germany

Die in diesem Artikel enthaltenen Bilder und sonstiges Drittmaterial unterliegen ebenfalls der genannten Creative Commons Lizenz, sofern sich aus der Abbildungslegende nichts anderes ergibt. Sofern das betreffende Material nicht unter der genannten Creative Commons Lizenz steht und die betreffende Handlung nicht nach gesetzlichen Vorschriften erlaubt ist, ist für die oben aufgeführten Weiterverwendungen des $\mathrm{Ma}$ terials die Einwilligung des jeweiligen Rechteinhabers einzuholen.

Weitere Details zur Lizenz entnehmen Sie bitte der Lizenzinformation auf http://creativecommons.org/ licenses/by/4.0/deed.de.

\section{Literatur}

1. Augustin M, Spehr C, Radtke MA et al (2014) German psoriasis registry PsoBest: objectives, methodology and baseline data. J Dtsch Dermatol Ges 12:48-57

2. Callizo J,Ziemssen F, Bertelmann Tetal (2019) Realworld data: ranibizumab treatment for retinal vein occlusion in the OCEAN study. Clin Ophthalmol 13:2167-2179

3. Ehlken C, Helms M, Bohringer D et al (2018) Association of treatment adherence with real-life VA outcomes in AMD, DME, and BRVO patients. Clin Ophthalmol 12:13-20

4. Herdman M, Gudex C, Lloyd A et al (2011) Development and preliminary testing of the new five-level version of EQ-5D (EQ-5D-5L). Qual Life Res 20:1727-1736
5. Holz FG, Johnson KT, Bauer-Steinhusen U et al (2020) Eine Untersuchung von Einflussfaktoren auf die Adhärenz von Patienten mit neovaskulärer altersabhängiger Makuladegeneration mithilfe des neu konzipierten Patientenfragebogens LAFIVT. Ophthalmologe 117:765-774

6. Holz FG, Tadayoni R, Beatty S et al (2015) Multicountry real-life experience of anti-vascular endothelial growth factor therapy for wet agerelated macular degeneration. $\mathrm{Br} \mathrm{J}$ Ophthalmol 99:220-226

7. HolzFG, Tadayoni R, Beatty Setal (2016) Key drivers of visual acuity gains in neovascular age-related macular degeneration in real life: findings from the AURA study. Br J Ophthalmol 100:1623-1628

8. KorobelnikJF, Do DV, Schmidt-Erfurth U etal (2014) Intravitreal afliberceptfor diabetic macular edema. Ophthalmology 121:2247-2254

9. Lommatzsch A, Eter N, Ehlken C et al (2020) Adhärenz bei der anti-VEGF Therapie - Überlegungen und praktische Empfehlungen. Ophthalmologe. https://doi.org/10.1007/s00347-020-01273-5

10. Massin P, Bandello F, Garweg JG et al (2010) Safety and efficacy of ranibizumab in diabetic macular edema (RESOLVEStudy): a 12-month, randomized, controlled, double-masked, multicenter phase II study. Diabetes Care 33:2399-2405

11. Mitchell P,BandelloF,Schmidt-ErfurthU etal (2011) The RESTORE study: ranibizumab monotherapy or combined with laser versus laser monotherapy for diabetic macular edema. Ophthalmology 118:615-625

12. Müller D, Augustin M, Banik N et al (2010) Memorandum Register für die Versorgungsforschung. Gesundheitswesen 72:824-839 
13. Neugebauer EA, Stausberg J (2016) Was Register leisten können und was nicht: Sicht der AG Register des Deutschen Netzwerks Versorgungsforschung e. V. (DNVF). Unfallchirurg 119:493-500

14. Nguyen NCl, FabroF, Ambresin A etal (2018) Factors associated with pain during intravitreal injection of anti-vascular endothelial growth factor. Eye (Lond) 32:1544-1546

15. Roth M, Holtmann C, Bohringer Det al (2020) Stand und Perspektiven registerbasierter Studien in der deutschen Augenheilkunde. Ophthalmologe 117:36-43

16. Segal O, Segal-Trivitz Y, Nemet AY et al (2016) Anxiety levels and perceived pain intensity during intravitreal injections. Acta Ophthalmol 94:203-204

17. Senra H, Balaskas K, Mahmoodi N et al (2017) Experience of anti-VEGF treatment and clinical levels of depression and anxiety in patients with wet age-related macular degeneration. Am J Ophthalmol 177:213-224

18. Sii S, Aspinall P, Borooah $S$ et al (2018) Exploring factors predicting changes in patients' expectations and psychosocial issues during the course of treatment with intravitreal injections for wet age-related macular degeneration. Eye (Lond) 32:673-678

19. Stausberg J, Maier B, Bestehorn K et al (2020) Memorandum Registerfür dieVersorgungsforschung: Update 2019. Gesundheitswesen 82:e39-e66

20. Stefanickova J, Cunha-Vaz J, Ulbig M et al (2018) A noninterventional study to monitor patients with diabetic macular oedema starting treatment with ranibizumab (POLARIS). Acta Ophthalmol 96:e942-e949
21. Wachtlin J, Eter N, Hasanbasic Z et al (2021) Importance of continuous treatment with intravitreal aflibercept injections in patients with neovascular age-related macular degeneration12-month post hoc analysis of the PERSEUS realworld evidence study. Graefes Arch Clin Exp Ophthalmol 259:601-611

22. Wenzel M, Scharrer A, Schayan K, Agostini H, Reinhard T (2020) Ambulante und stationäre Intraokularchirurgie 2019: Ergebnisse der aktuellen Umfrage von BDOC, BVA, DGIl und DOG. Ophthalmochirurgie 32:341-350

23. Westborg I, Granstam E, Rosso A et al (2017) Treatment for neovascular age-related macular degeneration in Sweden: outcomes at seven years in the Swedish macula register. Acta Ophthalmol 95:787-795

24. Wild B, Eckl A, Herzog W et al (2014) Assessing generalized anxiety disorder in elderly people using the GAD-7 and GAD-2 scales: results of a validation study. Am J Geriatr Psychiatry 22:1029-1038

25. Ziemssen F, Bertelmann T, Hufenbach U et al (2016) Verzögerung des Behandlungsbeginns um mehr als 2 Wochen. Relevanzfürmöglichen Visusgewinn nach Anti-VEGF-Therapie unter Real-Life-Bedingungen (Interimanalyse der prospektiven OCEANStudie). Ophthalmologe 113:143-115

\section{Bilder sagen mehr als Worte}

Wir suchen Ihre informativen und überraschenden Bilder!

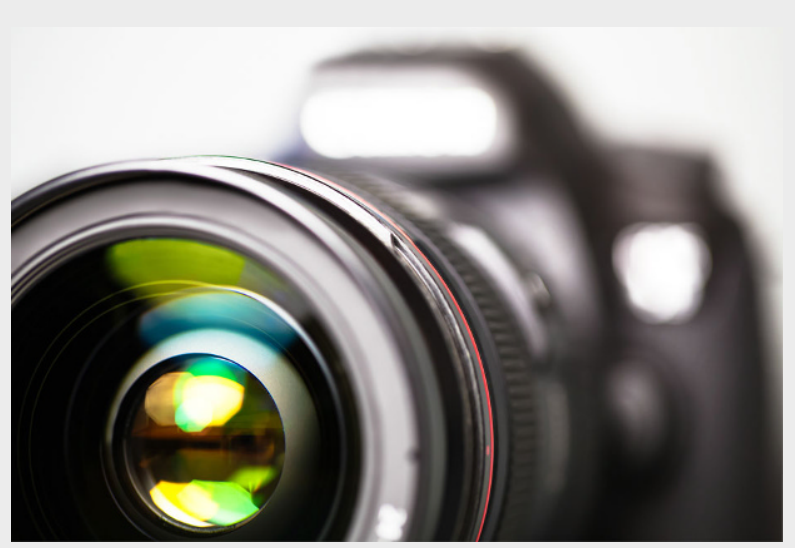

(c) Fotimmz / Fotolia

Verlag und Herausgeber*innenboard von Der Ophthalmologe laden Sie ein, die aufschlussreichsten Bilder aus Ihrem Alltag mit der Community zu teilen.
Schicken Sie uns Ihre Aufnahme oder eine klinisch-pathologische Korrelation mit einer prägnanten und aussagekräftigen Bildlegende.

Eine Auswahl der informativsten Schnappschüsse und klinisch-pathologischen Korrelationen werden dann, inklusive der Geschichte dahinter, in Der Ophthalmologe veröffentlicht. Wir freuen uns auf Ihre Beteiligung!

Hinweise zur Einreichung:

- 1 Abbildung bestehend aus max. sechs Einzelbildern (a-f); bei klinisch-pathologischer Korrelation unter Angabe von Färbung und Vergrößerung

- Aussagekräftiger Manuskript-Titel

- Bildlegende mit max. 2500 Zeichen inkl. Leerzeichen

- Max. 4 Autor*innen sowie vollständige Korrespondenzadresse

Senden Sie Ihre Bilder an:

Michal Meyer zu Tittingdorf

Managing Editor von Der Ophthalmologe

michal.meyerzutittingdorf@springer.com 